\title{
The inhibition of heme oxygenase-1 enhances the chemosensitivity and suppresses the proliferation of pancreatic cancer cells through the SHH signaling pathway
}

\author{
LIANG HAN $^{1 *}$, JIE JIANG ${ }^{2 *}$, QINGYONG MA ${ }^{1}$, ZHENG WU $^{1}$ and ZHENG WANG ${ }^{1}$ \\ ${ }^{1}$ Department of Hepatobiliary Surgery, The First Affiliated Hospital, Xi'an Jiaotong University, \\ Xi'an, Shaanxi 710061; ${ }^{2}$ Department of Medical Oncology, \\ Shaanxi Provincial People's Hospital, Xi'an, Shaanxi 710068, P.R. China
}

Received October 6, 2017; Accepted February 5, 2018

DOI: 10.3892/ijo.2018.4363

\begin{abstract}
Pancreatic cancer (PC) is a type of cancer associated with a high fatality rate due to a poor prognosis and resistance to treatment. Heme oxygenase-1 (HO-1) is significantly overexpressed in a number of types of cancer and seems to play an important role in cancer progression. In this study, we examined the potential effects of HO-1 on PC cell proliferation and sensivity to gemcitabine (Gem). Furthermore, the role of the sonic hedgehog (SHH) signaling pathway in the regulatory effects of HO-1 on PC progression were examined. For this purpose, the expression of HO-1 was examined in cultured PC cells by real-time PCR, western blot analysis and immunofluorescence. Transfection with small interfering RNA against HO-1 or an overexpression plasmid were used to regulate the expression of HO-1 in the MIA PaCa-2 and PANC-1 cell lines. Cell proliferation was examined by MTT assays in response to the different treatments. The results revealed that HO-1 expression differed significantly in the different PC cells. The overexpression of HO-1 induced PC cell proliferation and the inhibition of HO-1 decreased the cell proliferative ability. Furthermore, HO-1 activated the SHH signaling pathway in the PC cells. In addition, the SHH signaling pathway was found to play a role in HO-1-induced PC cell proliferation. The inhibition of HO-1 enhanced the responsiveness of PC cells to Gem and Gem was found to regulate the expression of HO-1 and the activation of the SHH pathway. On the whole, our findings indicate that $\mathrm{HO}-1$ overexpression in PC cells may be responsible for the increased cell proliferation and the resistance to anticancer therapy. Furthermore, the SHH signaling
\end{abstract}

Correspondence to: Dr Zheng Wang, Department of Hepatobiliary Surgery, The First Affiliated Hospital, Xi'an Jiaotong University, 277 West Yanta Road, Xi'an, Shaanxi 710061, P.R. China

E-mail: hanliangxjtu@163.com

*Co-first authorship

Key words: pancreatic cancer, heme oxygenase-1, sonic hedgehog, gemcitabine pathway, the activation of which was initiated by HO-1, may be one of the endogenous mechanisms in this process. Our data shed light into the association between $\mathrm{HO}-1$ and $\mathrm{SHH}$ in PC cells, and may aid in the development of novel therapeutic targets for the treatment of patients with PC.

\section{Introduction}

Pancreatic cancer (PC) accounts for the majority of pancreatic malignancies, and is a devastating disease characterized by a late diagnosis, poor prognosis and resistance to chemotherapy $(1,2)$. Many patients have advanced unresectable tumors at the time of diagnosis. The effectiveness of current chemotherapeutic regimens and radiotherapy is not sufficient. Therefore, additional treatment options for patients with advanced PC are urgently required.

Heme oxygenase (HO)- 1 is the inducible isoform of the three heme oxygenases (HO-1, HO-2 and HO-3) that catalyze the degradation of heme into biliverdin, carbon monoxide (CO) and free iron (3). HO-1 and its metabolites affect inflammatory and apoptotic properties. Furthermore, HO-1 regulates cancer cell proliferation and angiogenesis (4). In a recent studies, HO-1 expression and activity was found in various tumors, such as prostate cancer (5), lung cancer (6) and colon cancer (7). Data from previous studies have also shown that HO-1 inhibits the proliferation of pancreatic stellate cells (8), and that HO-1 affects the responsiveness of PC to anticancer treatment (9).

The sonic hedgehog $(\mathrm{SHH})$ signaling pathway plays a fundamental role in organ development and differentiation. $\mathrm{SHH}$, which is a secreted protein, binds to Patched (PTCH) allows smoothened (SMO) to activate downstream factors, such as Gli1 or Gli2, and regulate target gene expression. The SHH signaling pathway, a major regulator of cancer cell proliferation and differentiation, contributes to metastasis (10). In PC cells, the SHH signaling pathway has been shown to be involved in pain associated with PC (11), and in the development of pancreatic fibrosis (12).

Gemcitabine (Gem) is one of the most important chemotherapeutic agents used in the treatment of patients with PC. Although this drug is effective, its cytotoxic effects and drug resistance limit its application and curative effects, leading to 
poor patient outcomes. An increasing amount of research has been carried out with an aim to determine the mechanisms responsible for sensitivity and resistance to $\operatorname{Gem}(9,13,14)$. Furthermore, the SHH signaling pathway is partly involved in the resistance of PC cells to Gem $(15,16)$.

The above-mentioned findings suggest that $\mathrm{HO}-1$ and $\mathrm{SHH}$ signaling may play a crucial role in PC progression and sensitivity to chemotherapy. In this study, we examined the potential effects of HO-1 on PC cell proliferation and sensitivity to Gem. Furthermore, the role of the SHH signaling pathway in the mechanisms of HO-1-induced PC cell proliferation were investigated.

\section{Materials and methods}

Cell culture and reagents. The established human $\mathrm{PC}$ cell lines, BxPc-3, MIA PaCa-2, PANC-1 and SW1990, were obtained from the American Type Culture Collection (ATCC, Manassas, VA, USA) and cultured in DMEM supplemented with $10 \%$ fetal bovine serum (FBS) and 1\% antibiotic/antimycotic (Life Technologies, Carlsbad, CA, USA). The cells were maintained at $37^{\circ} \mathrm{C}$ in a humidified $5 \% \mathrm{CO}_{2}$ atmosphere. Antibodies against SHH (ab53281), SMO (ab5694), PTCH (ab53715), Gli1 (ab49314), Gli2 (ab26056), GAPDH (ab8245) and HO-1 (ab13248) were purchased from Abcam (Cambridge, MA, USA). Recombinant SHH, and zinc protoporphyrin IX (ZnPPIX) were obtained from R\&D Systems (Minneapolis, MN, USA).

Real-time PCR. Total RNA was extracted from the cells using TRIzol reagent (Invitrogen, Carlsbad, CA, USA), and cDNA was synthesized using a Prime Script RT reagent kit (Takara, Dalian, China). The real-time PCR experiments were conducted on an iQ5 Multicolor Real-Time PCR Detection System (Bio-Rad Laboratories Inc., Hercules, CA, USA) using SYBRGreen Real-time PCR Master Mix (Takara). Amplification was carried out as follows: denaturation at $94^{\circ} \mathrm{C}$ for $3 \mathrm{~min}, 35$ cycles of $94^{\circ} \mathrm{C}$ for $30 \mathrm{sec}, 58^{\circ} \mathrm{C}$ for $30 \mathrm{sec}$, and $72^{\circ} \mathrm{C}$ for $35 \mathrm{sec}$. The sequences of the primers used were as follows: HO-1 forward, 5'-CAG GCA GAG GGT GAT AGA AGA GG-3' and reverse, 5'-CTG GGA GCG GGT GTT GAG TG-3'; and GAPDH forward, 5'-ACCACAGTCCATGCCATCAC-3' and reverse, 5'-TCCACCACCCTGTTGCTGTA-3'. The expression of the target gene was calculated using the $2^{-\Delta \Delta \mathrm{Cq}}$ method (17). All experiments were repeated independently 3 times.

Western blot analysis. Protein was extracted from the cells using lysis buffer [50 mM Tris ( $\mathrm{pH} 7.5$ ), $150 \mathrm{mM} \mathrm{NaCl}, 1 \%$ NP-40, $0.5 \%$ sodium deoxycholate, $1 \mathrm{mM}$ EDTA, and $0.1 \%$ SDS] containing a protease inhibitor cocktail (Sigma-Aldrich, St. Louis, MO, USA), and protein concentrations were measured by DC Protein Assay (Bio-Rad Laboratories Inc.). Following separation on $7.5 \%$ SDS-polyacrylamide gels, the proteins $(20 \mu \mathrm{l})$ were transferred onto nitrocellulose membranes (Millipore, Billerica, MA, USA), which were then incubated with the primary antibodies $(1: 1,000)$ at $4^{\circ} \mathrm{C}$ overnight. After washing 3 times with TBST, the membranes were incubated with horseradish peroxidase-conjugated secondary antibodies (rabbit, 1:1,000, ab191866; mouse, 1:1000, ab193651; Abcam) for $1 \mathrm{~h}$. Immunoreactive bands were visualized using an enhanced chemiluminescence kit (Millipore). Quantitative analysis was performed using Image-Pro Plus 6.0 software (Media Cybernetics, Inc., Rockville, MD, USA). The relative protein expression levels were normalized to GAPDH. All experiments were repeated independently 3 times.

MTT assay. Cell proliferation rates were measured by MTT assays. Briefly, the cells were seeded in 96-well plates at a density of $1 \times 10^{4}$ cells per well and incubated overnight in medium containing $10 \%$ FBS. The DMSO concentration was adjusted to $0.4 \%$. The cells incubated in serum-free medium were used as the control group. Following incubation for 24,48 and $72 \mathrm{~h}$ at $37^{\circ} \mathrm{C}, 20 \mu \mathrm{l}$ of MTT solution $[5 \mathrm{mg} / \mathrm{ml}$ in phosphate-buffered saline (PBS)] were added to each well, and the cells were incubated for an additional $4 \mathrm{~h}$ at $37^{\circ} \mathrm{C}$. Subsequently, $100 \mu 1 \mathrm{DMSO}$ were added to each well at $37^{\circ} \mathrm{C}$. The optical density (OD) value was determined using a spectrophotometer (Bio-Rad Laboratories Inc.) at $490 \mathrm{~nm}$. The proliferation rate was defined as OD (cell plate)/OD (blank plate). All experiments were repeated independently 3 times.

Immunofluorescence staining. HO-1 localization in PC cells was examined by immunofluorescence. The prepared cells were washed 3 times with PBS and then fixed with $100 \mathrm{ml}$ $4 \%$ paraformaldehyde in PBS. The cells were permeabilized in blocking buffer $(0.1 \%$ Triton $\mathrm{X}-100$ or $0.1-0.5 \%$ saponin, $10 \%$ NGS, $100 \mathrm{mM}$ PBS, $\mathrm{pH}$ 7.4) for $1 \mathrm{~h}$ at room temperature and then incubated with primary antibody to HO-1 overnight at $4^{\circ} \mathrm{C}$. The following day, the cells were washed and incubated with FITC-conjugated goat anti-mouse IgG (green fluorescence; 1:500, anti-mouse, \#115165003; Jackson ImmunoResearch, West Grove, PA, USA) for $1 \mathrm{~h}$ at room temperature. The cell nuclei were stained with 4',6-diamidino2-phenylindole (DAPI; \#0100-20, SouthernBiotech, Birmingham, AL, USA) for $10 \mathrm{~min}$. After washing with PBS 3 times, cells were blocked for $5 \mathrm{~min}$. As a negative control, the primary antibody was substituted with antibody diluent. All experiments were repeated independently 3 times.

Transfection with HO-1 small interfering RNA (siRNA) and plasmid construction. HO-1 expression was specifically suppressed by the introduction of 21-nucleotide duplex siRNA. The sequences of the ribonucleotides used were as follows: 5'-rGACUGCGUUCCUGCUCAACdTdT-3' and 5'-rGUUGA GCAGGAACGCAGUCdTdT-3' (Ambion, Inc., Austin, TX, USA). The cells seeded into small dishes were transfected with $100 \mathrm{nM}$ siRNA using Lipofectamine 2000 (Invitrogen) according to the manufacturer's instructions. Negative control siRNA (Ambion Inc.) was used as a negative control. In addition, plasmids for the overexpression of HO-1 were constructed according to manufacturer's instructions (Roche, Penzberg, Germany). The untransfected cells used in the experiment were cultured under normal conditions. All experiments were repeated independently 3 times.

Drug treatments. Recombinant SHH (\#CYT597; ProSpec-Tany TechnoGene Ltd., Ness-Ziona, Israel) was applied to the cultured PC cells at $5 \mathrm{mg} / \mathrm{ml}$. In addition, cyclopamine (Cyc) (\#4449518; ApexBio, Houston, TX, USA), a SHH pathway inhibitor, was diluted to $18 \mu \mathrm{g} / \mathrm{ml}$ in PC cell medium. The compound, ZnPPIX, 
A

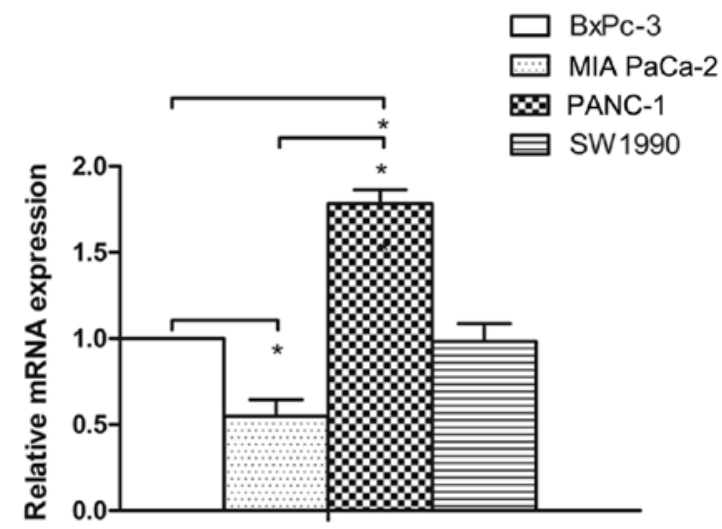

B
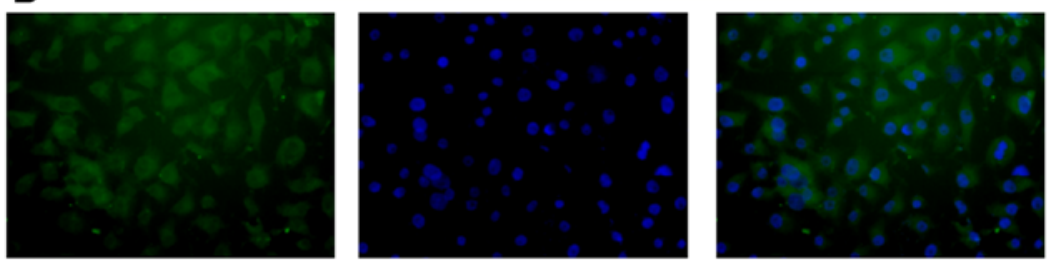

PANC-1

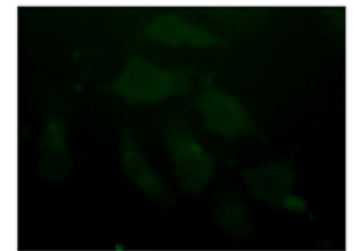

Green

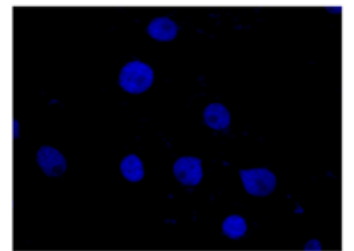

DAPI

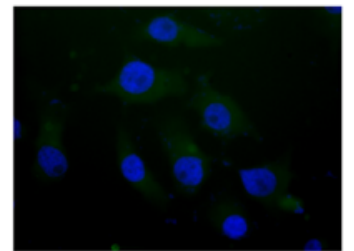

Merge
MIA PaCa-2

Figure 1. HO-1 is overexpressed in PC cells. (A) HO-1 mRNA expression was determined by real-time PCR. The graph shows the mRNA expression of HO-1 in different PC cells. On average, the mRNA expression HO-1 was higher in the PANC-1 cells compared with the other groups. (B) Immunofluorescence staining of HO-1 in the MIA PaCa-2 and PANC-1 cell lines.

an inhibitor of HO-1, was used at $50 \mu \mathrm{M}$ (18). Gem was applied according to the PC cell line-specific EC50 $(15 \mu \mathrm{g} / \mathrm{ml}$ for the MIA PaCa- 2 cells and $100 \mu \mathrm{g} / \mathrm{ml}$ for the PANC-1 cells $(9,19)$.

Statistical analysis. The analyses of the results was carried out using the SPSS statistical software package (version 13.0). The significance of the data was determined using a Student's t-test or one-way analysis of variance (ANOVA), followed by the Bonferroni post hoc test, where applicable. A value of $\mathrm{P}<0.05$ was considered to indicate a statistically significant difference. All results are expressed as the means \pm SD. All the experiments were repeated 3 times.

\section{Results}

HO-1 is overexpressed in PC cells. To determine whether HO-1 plays an important role in the progression of PC, the mRNA expression of HO-1 was identified by real-time PCR in the BxPc-3, MIA PaCa-2, PANC-1 and SW1990 cells human PC cells (Fig. 1A). The results revealed that HO-1 mRNA expression differed significantly in the different PC cells. On average, a 3-fold upregulation of HO-1 mRNA expression was observed in the PANC-1 cells compared with the MIA PaCa- 2 cells $(\mathrm{P}<0.05)$.

Thus, we selected the MIA PaCa- 2 and PANC-1 cell lines for use in the following experiments due to the fact that they expressed low and high levels of HO-1, respectively. The protein expression of HO-1 was examined by the immunofluorescence staining of the MIA PaCa-2 and PANC-1 cell lines (Fig. 1B). As a negative control, the primary antibody was substituted with antibody diluent (data not shown). The expression of HO-1 in the PC cells provided the basis for our subsequent research.

Expression of HO-1 induces $P C$ cell proliferation and inhibition of $\mathrm{HO}-1$ reduces the proliferation ability. To examine the effects of HO-1 on PC cell growth, the PANC-1 (high HO-1 expression) and $\mathrm{MIA} \mathrm{PaCa}-2$ cells (low $\mathrm{HO}-1$ expression) were used for transfection. The PANC-1 cells were transfected with HO-1 siRNA, and the MIA PaCa-2cells were transfected with HO-1 expression plasmids. At $12 \mathrm{~h}$ after transfection, the transfection efficiencies were verified by real-time PCR (Fig. 2A and B) and western blot analysis (Fig. 2C). Cell proliferation in response to $\mathrm{HO}-1$ stimulation was measured by MTT assays at 24, 48 and $72 \mathrm{~h}$ following transfection or treatment with ZnPPIX. Our results revealed that the PANC-1 cells transfected with siRNA (PANC-1-si-HO-1) exhibited a decreased rate of proliferation compared with the control group; ZnPPIX was used as a positive control and treatment with ZnPPIX also decreased cell proliferation $(\mathrm{P}<0.05)$ (Fig. 2D). On the contrary, the MIA PaCa-2 cells transfected with the HO-1 expression plasmid exhibited an increased proliferation rate $(\mathrm{P}<0.05)$ (Fig. 2E). 

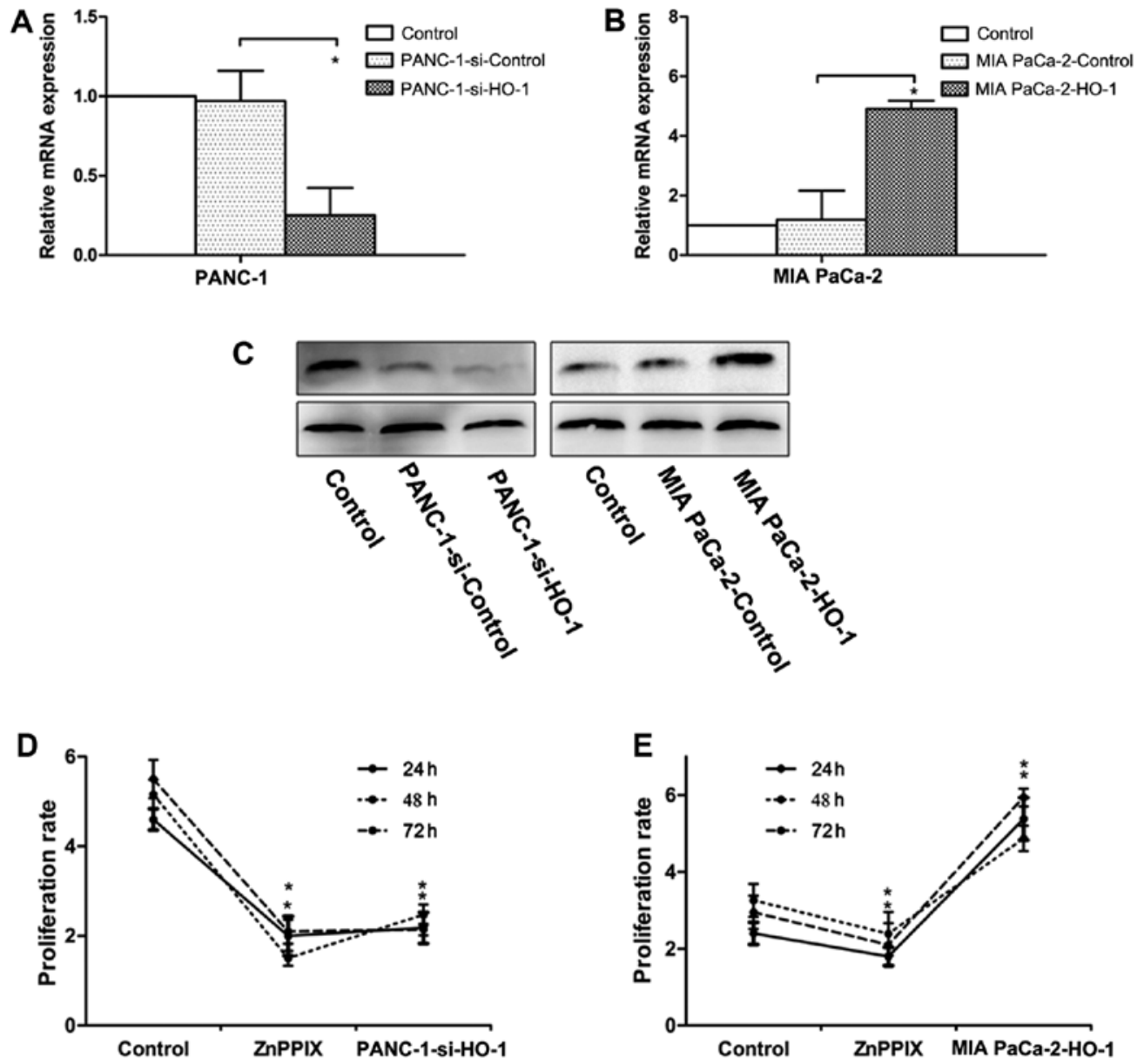

Figure 2. HO-1 induces PC cell proliferation and the inhibition of HO-1 suppressess the proliferative ability. (A and B) The transfection efficiencies were verified by real-time PCR. PANC-1 cells were transfected with HO-1 siRNA. MIA PaCa-2 cells were transfected with HO-1 expression plasmid. (C) The transfection efficiencies were examined by western blot analysis, which revealed similar results as those obtained by real-time PCR. (D and E) The effects of HO-1 on PC cell proliferation were determined by MTT assay. Our data revealed that the downregulation of HO-1 led to the suppression of the proliferation of PANC-1 cells $(\mathrm{P}<0.05)$, and the upregulation of HO-1 increased the proliferation of MIA PaCa-2 cells. ZnPPIX, a HO-1 activity inhibitor, was used as a positive control; "P<0.05 compared with the control.

HO-1 induces SHH signaling pathway activation in PC cells. To examine the effects of HO-1 on the SHH signaling pathway in the PC cells, we examined the expression of molecules associated with the SHH signaling pathway in the PC cells under various treatment conditions by western blot analysis (Fig. 3A). Based on this analysis, the PANC-1 cells transfected with siRNA against HO-1 (PANC-1-si-HO-1) exhibited a decreased expression of SHH and Glil compared with the untransfected PANC-1 cells; similar results were obtained with the PANC-1 cells treated with the positive control, ZnPPIX ( $\mathrm{P}<0.050$ (Fig. 3B). We also found that the MIA PaCa-2 cells transfected with the HO-1 expression plasmid (MIA PaCa-2-HO-1) exhibited an increased expression of SHH and Gli1 ( $\mathrm{P}<0.050$ (Fig. 3B). However, no marked differences were observed in the expression of PTCH and SMO the cell groups $(\mathrm{P}>0.05)$. Thus, these results indicate that HO-1 activates the SHH signaling pathway in PC cells.

SHH signaling pathway plays role in HO-1-induced PC cell proliferation. To determine whether the SHH pathway is involved in the high proliferation rate induced by HO-1, as shown in Fig. 4, the PANC-1 and MIA PaCa-2 cells were treated with Cyc, which is a $\mathrm{SHH}$ signaling pathway inhibitor.
The results revealed the dose-dependent effects of Cyc on cell proliferation (data not shown). In addition, treatment of the PANC-1 cells with Cyc in combination with transfection with the HO-1 overexpression vector significantly decreased the PANC-1 cell proliferative ability in comparison with the controls or the other intervention groups $(\mathrm{P}<0.05)$ (Fig. 4A). Additionally, as regards the MIA PaCa- 2 cells, the results revealed that treatment of these cells with Cyc inhibited the proliferation, compared with the control group and the MIA PaCa-2 cells transfected with the expression vector and not treated with Cyc $(\mathrm{P}<0.05)$ (Fig. 4B). Thus, HO-1 was found to activate the SHH signaling pathway in PC cells; thus, this suggests that the activation of the SHH signaling pathway is involved in HO-1-induced PC cell proliferation. However, the mechanisms through which the SHH signaling pathway is activated by HO-1 remain unknown. Whether the activation of the SHH signaling pathway occurs through an autocrine manner or through other mechanisms, is worthy of investigation. To clarify this, we used exogenous SHH to treat the PC cells. The results revealed that differences were only found between the cells not treated with SHH and those that were treated with SHH in the control group; no significant changes in the cell proliferative ability were 
A
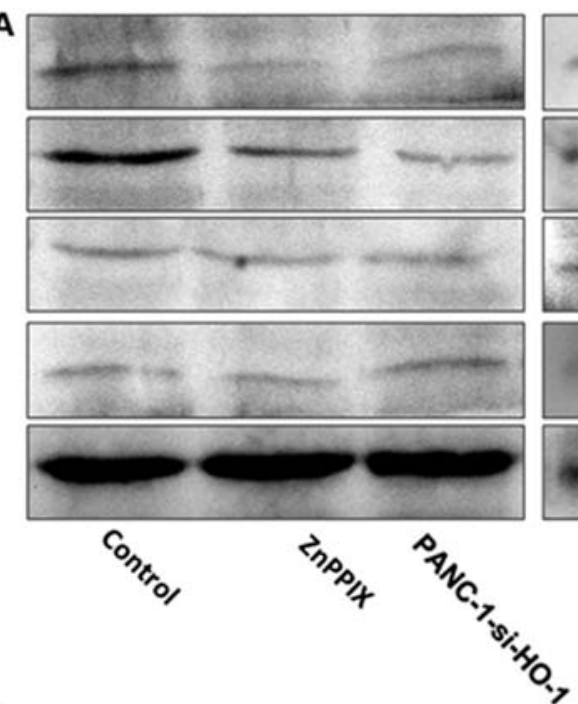

B

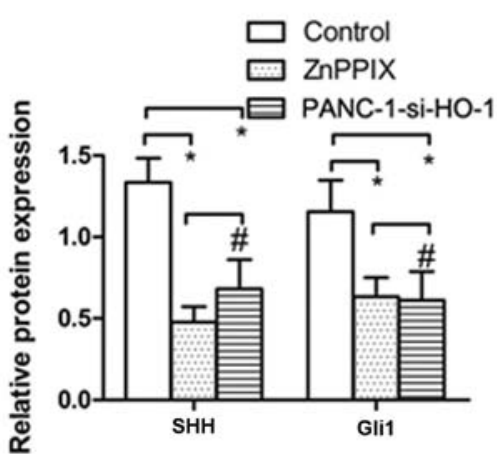

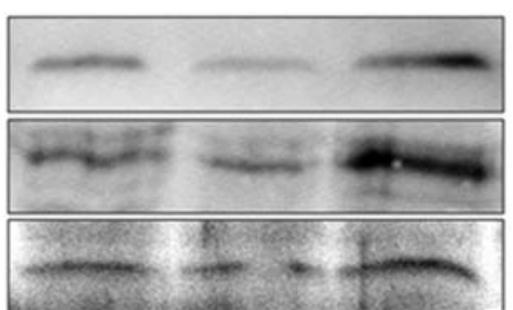

SHH

Gli1

PTCH
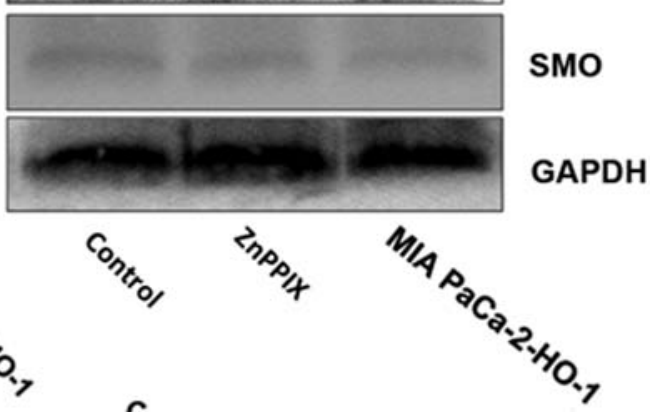

C

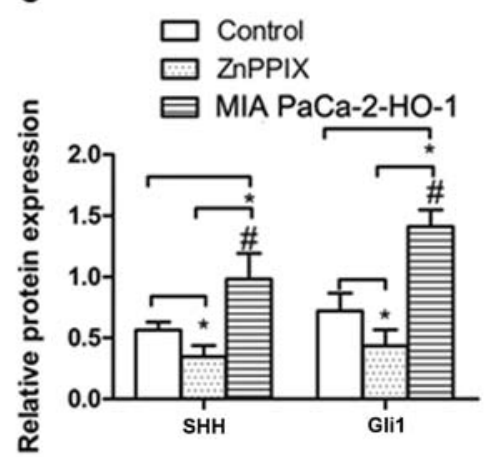

Figure 3. Effect of HO-1 on SHH signaling pathway activation in PC cells. (A) The effect of HO-1 on the SHH signaling pathway was examined by western blot analysis in PC cells. (B and C) The PANC-1 cells transfected with siRNA against HO-1 (PANC-1-si-HO-1) and those treated with ZnPPIX exhibited a decreased expression of SHH signaling molecules (SHH and Gli1); however, the MIA PaCa-2 cells transfected with an HO-1 expression plasmid (MIA $\mathrm{PaCa}-2-\mathrm{HO}-1)$ exhibited an increased expression of SHH and Gli1; ${ }^{*} \mathrm{P}<0.05$ compared with the control; ${ }^{*} \mathrm{P}<0.05$ compared with ZnPPIX treatment.

observed in the other 3 intervention groups in both cell lines. $(\mathrm{P}>0.05)$ (Fig. 4C and D).

Inhibition of HO-1 enhances the responsiveness of $P C$ cells to Gem. To determine whether the chemoresistance of PC cells is due to $\mathrm{HO}-1$, we examined $\mathrm{PC}$ cell proliferation when the MIA PaCa-2 and PANC-1 cells were transfected with either HO-1 siRNA or the HO-1 expression plasmid and treated with Gem (EC50 dose). The results of the dose-dependent experiments of Gem treatment are shown in Fig. 5A and B). We found that the PANC-1 cells transfected with HO-1 siRNA (PANC-1-si-HO-1) and treated with Gem exhibited a decreased proliferative ability $(\mathrm{P}<0.05)$ (Fig. 5C). By contrast, MIA PaCa-2 cells transfected with the HO-1 expression vector and treated with Gem exhibited a greater proliferative ability than the MIA $\mathrm{PaCa}-2$ cells treated with Gem alone $(\mathrm{P}<0.05)$ (Fig. 5D). Thus, a decreased proliferative ability was observed after $\mathrm{HO}-1$ inhibition and treatment with Gem.

HO-1 and SHH pathway activation can be induced by Gem in $P C$ cells chemosensitivity. To determine whether HO-1 affects the chemosensitivity of PC cells, we treated the PC cells with an EC50 dose of Gem for $24 \mathrm{~h}$. The quantification data from realtime PCR revealed that Gem increased the mRNA expression of HO-1 in the PANC-1 and MIA PaCa-2 cells compared with the untreated controls $(\mathrm{P}<0.05)$ (Fig. 6A). Furthermore, the cells treated with Gem exhibited an increased protein expression of HO-1 in both cell lines $(\mathrm{P}<0.05)$ (Fig. 6B). To determine whether Gem is responsible for the activation of the $\mathrm{SHH}$ pathway, we examined the expression of $\mathrm{SHH}$ and Gli1 in the PC cells treated with Gem. Treatment with Gem resulted in an increase in the expression of Gli1 in the PANC-1 and MIA PaCa-2 cells $(\mathrm{P}<0.05)$ (Fig. 6C). However, no significant induction of SHH expression observed in both cell lines $(\mathrm{P}>0.05)$.

\section{Discussion}

$\mathrm{PC}$ is one of the most lethal types of cancer. While the mortality rates are improving as with other types of cancer, the clinical outcomes of patients with $\mathrm{PC}$ remains abysmally poor (1). $\mathrm{HO}-1$ is the rate-limiting enzyme involed in the degradation of heme into carbon monoxide, iron and biliverdin. HO-1 regulates cell proliferation (4) and accelerates PC angiogenesis (20). The proliferation of pancreatic stellate cells, which were the key process in PC development has been shown to be inhibited by HO-1 via the extracellular signal-regulated kinase (ERK)1/2 pathway (8). Furthermore, HO-1 functions as a mediator of the therapeutic effects (21). The present study 

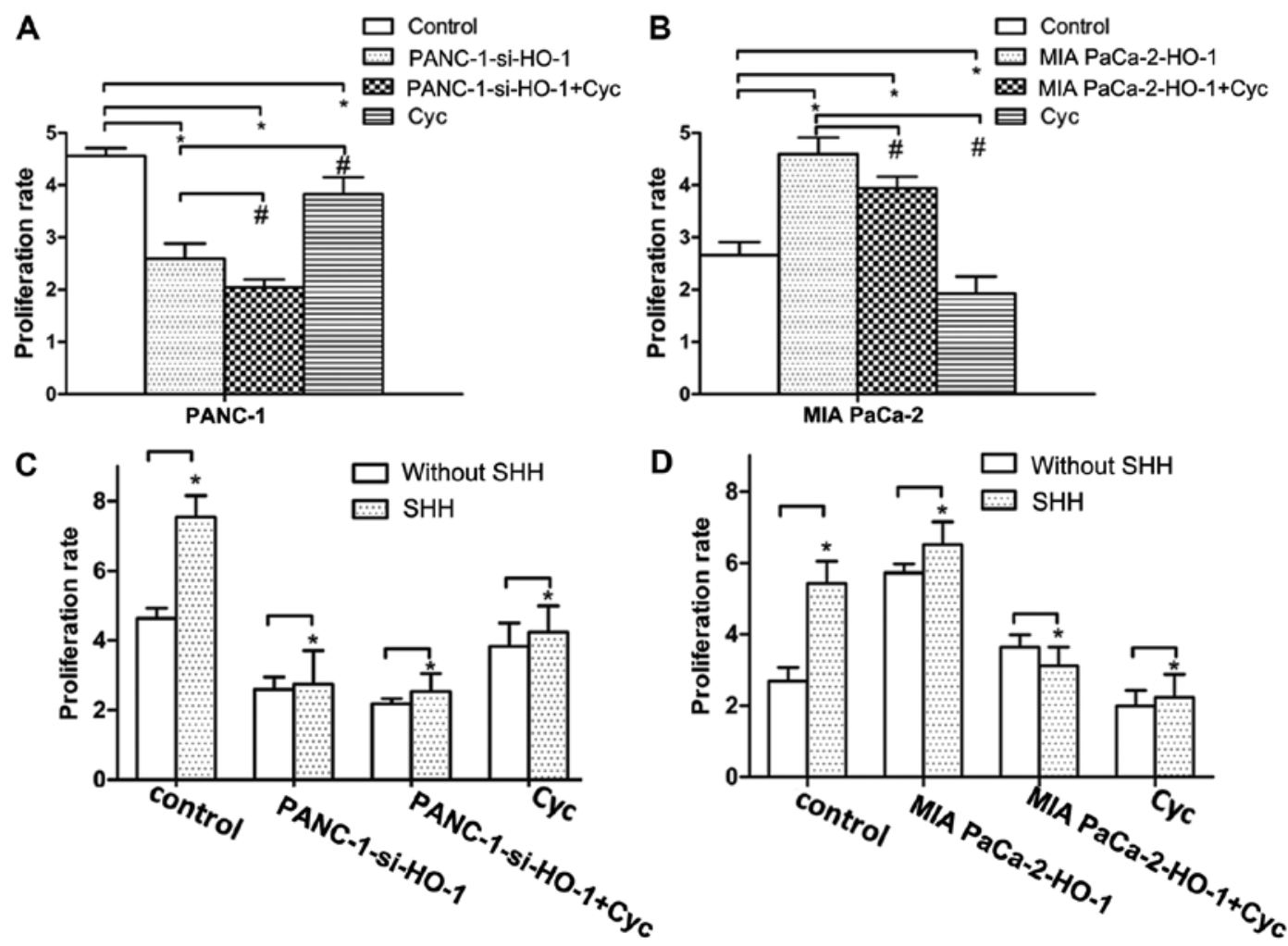

Figure 4. Effect of cyclopamine (Cyc) or SHH on HO-1-induced PC cell proliferation. (A and B) The PC cells were treated with Cyc. The results revealed that treatment with Cyc combined with the downregulation of HO-1 in PC cells by siRNA significantly decreased the cell proliferative ability; ${ }^{*} \mathrm{P}<0.05$ compared with the control; " $\mathrm{P}<0.05$ compared with the transfection group. (C and D) Exogenous SHH was used to treat the PC cells. The results revealed that, apart from the control group, no change in the proliferative ability was observed among the 3 intervention groups $(\mathrm{P}>0.05)$. Changes were only observed between the cells treated with or without SHH ( $(\mathrm{P}<0.05)$.
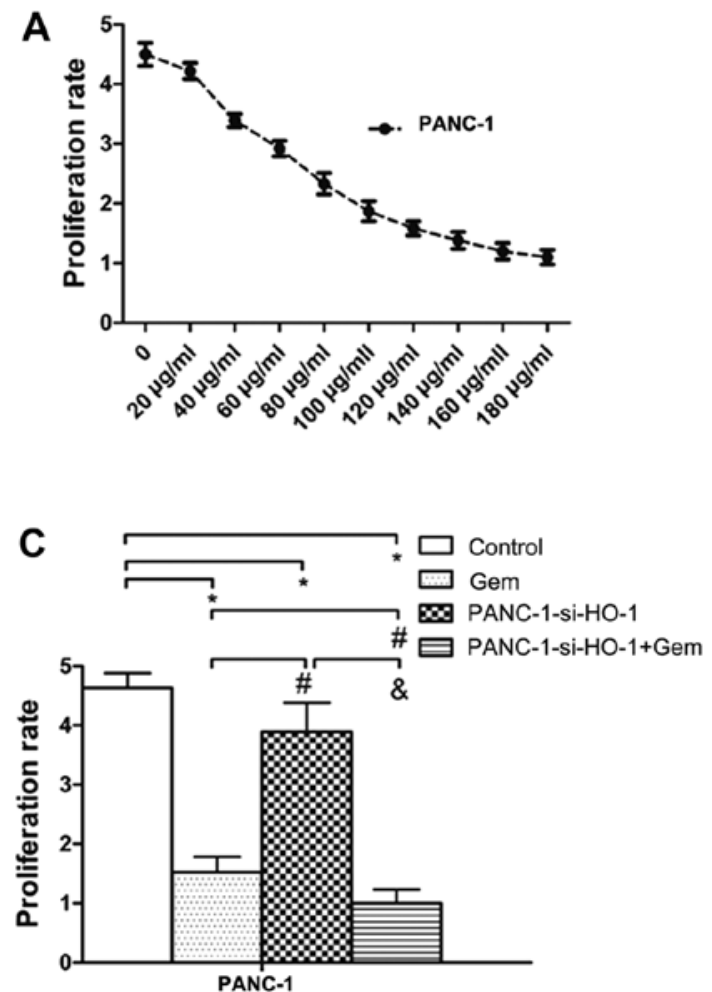
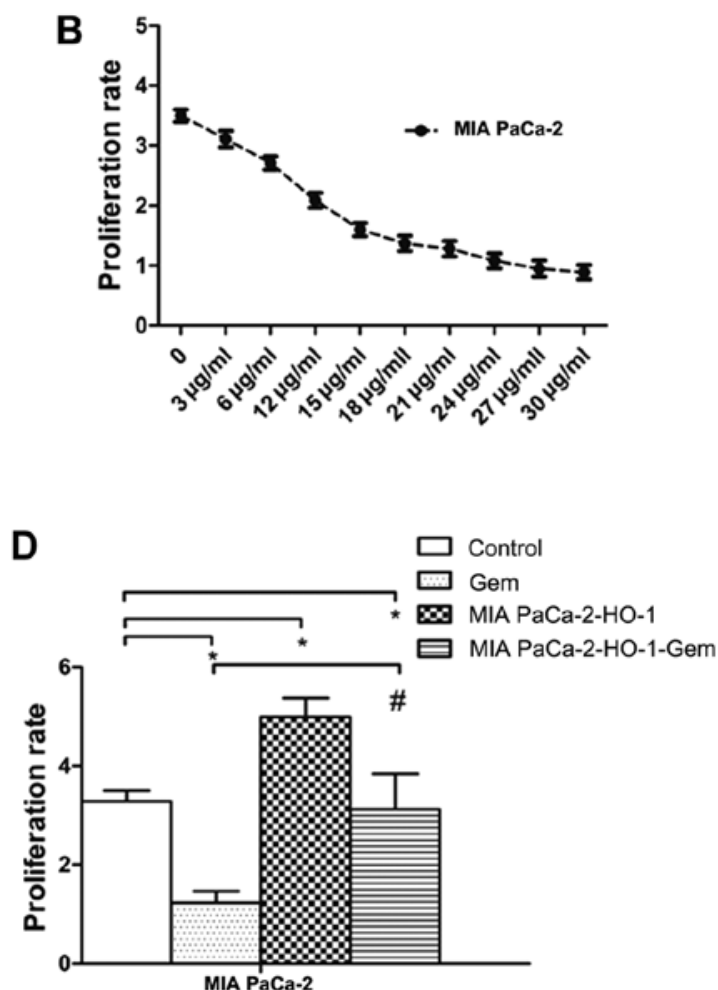

Figure 5. Downregulation of HO-1 expression sensitizes PC cells to Gem. (A and B) The PC cells were treated with gemcitabine (Gem), and the data revealed the dose-dependent effects. (C) The data revealed that the downregulation of HO-1 using siRNA sensitized the PC cells to chemotherapy and decreased cell proliferation. (D) For the MIA PaCa-2 transfected with the HO-1 expression plasmid (MIA PaCa-2-HO-1), the sensitivity to Gem was reduced and the cancer cells had an increased proliferative ability; ${ }^{*} \mathrm{P}<0.05$ compared with the control; ${ }^{*} \mathrm{P}<0.05$ compared with the Gem treatment group; ${ }^{\text {\& }} \mathrm{P}<0.05$ compared with the PANC-1-si-HO-1 group (PANC-1 cells transfected with siRNA). 
A

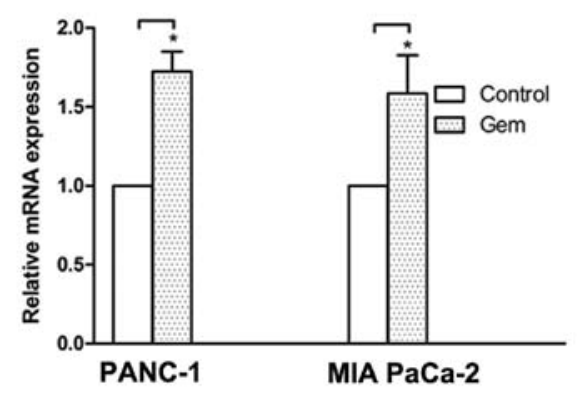

B

PANC-1

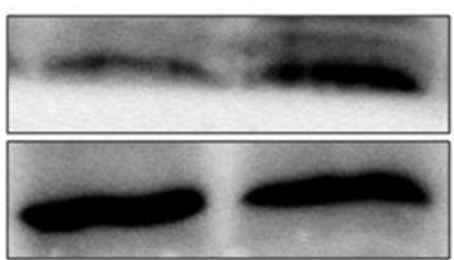

Control

C

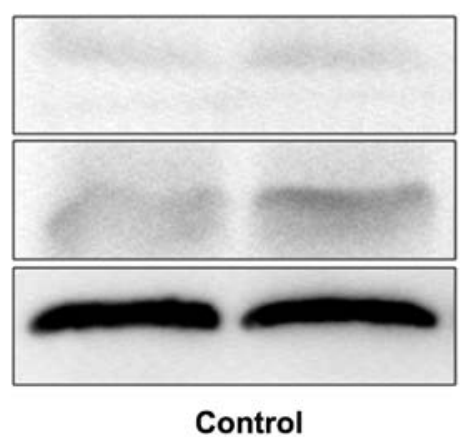

PANC-1

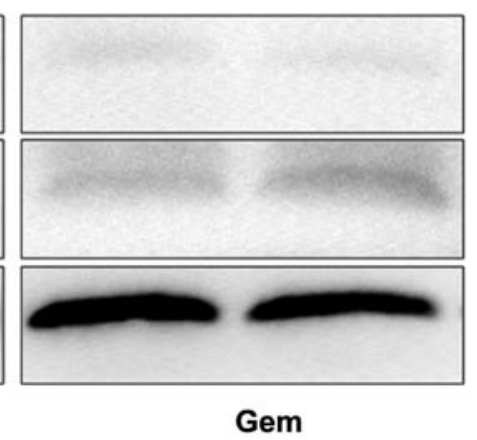

HO-1

GAPDH

SHH

Gli-1

GAPDH

Figure 6. Effect of gemcitabine (Gem) on HO-1 expression and SHH signaling pathway activation. (A) The effect of Gem on HO-1 mRNA expression was verified by real-time PCR. The results revealed that Gem increased the mRNA expression of HO- 1 in the PANC-1 and MIA PaCa- 2 cells ( $\mathrm{P}<0.05$ compared with the control). (B) Western blot analysis of HO-1 protein expression revealed that Gem induced HO-1 expression compared with the control ( $<<0.05$ ). (C) The levels of SHH signaling pathway were assessed by western blot analysis. Gem resulted in the upregulation of Gli1 in the PANC-1 and MIA PaCa-2 cells $(\mathrm{P}<0.05)$. However, the protein levels of SHH remained unaltered.

focused on the association between $\mathrm{HO}-1$ and $\mathrm{SHH}$ in $\mathrm{PC}$. As reported previously, $\mathrm{SHH}$ regulates pancreatic fibrosis and cancer cell proliferation and differentiation $(22,23)$. Gem is one of the most important chemotherapeutic agents used in the treatment of the majority of patients with PC $(13,24)$. Over the past several years, first-line therapy with Gem has boosted the median overall survival (OS) of patients with PC $(25,26)$. However, the effectiveness of Gem is limited due to drug resistance in patients with PC. Thus far, the mechanisms of resistance have been tested for mutations in several genes; however, the mechanisms involved are not yet very clear $(27,28)$. Some studies have reported the correlation between resistance to Gem and SHH signaling $(15,29)$.

The main results of this study indicate that the upregulation of HO-1 expression promotes PC cell proliferation, and that the inhibition of HO-1 enhances the responsiveness of $\mathrm{PC}$ cells to Gem. In addition, our data indicate that the $\mathrm{SHH}$ signaling pathway plays an important role in this process.

This study demonstrated that the expression levels of HO-1 differed among the PC cell lines. Furthermore, the MIA PaCa-2 and PANC-1 cell lines were selected for use in the following experiments due to the fact that they expressed low and high levels of HO-1, respectively. We suppressed HO-1 expression by siRNA transfection and evaluated the growth behavior of the PANC-1 cells. The analysis revealed a significant inhibitory effect on the proliferation of the cells expressing low levels of HO-1; similar results were obtained with cells treated with the HO-1 activity inhibitor, ZnPPIX. Moreover, we found a significantly increased proliferation rate in the MIA PaCa-2 cells expressing high HO-1 expression levels by transfection with an HO-1 expression plasmid in comparison to the control group. Thus, the expression of HO-1 induced PC cell proliferation and the inhibition of HO-1 suppresses PC cell proliferation.

More importantly, we examined the potential mechanisms responsible for $\mathrm{HO}-1$-induced $\mathrm{PC}$ cell proliferation. We examined the effect of HO-1 on the SHH signaling pathway in PC cells. The results revealed that the PANC-1 cells transfected with siRNA against HO-1 exhibited a decreased expression of SHH and Glil compared with the untransfected PANC-1 cells. However, the MIA PaCa-2 cells expressing high levels of $\mathrm{HO}-1$ by transfection with an HO-1 expression plasmid exhibited 
an increased expression of SHH and Gli1. No marked differences were observed in the levels of PTCH and SMO. In our study, it was evident that HO-1 activated the SHH signaling pathway in PC cells. Our results supported by evidence from a previous study on the effects of $\mathrm{HO}-1$ upregulation on the $\mathrm{SHH}$ signaling pathway (30).

In following experiments, treatment with Cyc significantly decreased the proliferative ability when the expression of HO-1 was decreased by siRNA. Thus, it was proven that the SHH signaling pathway also acts as a downstream target of HO-1 in PC cells. Moreover, PC cells were treated with exogenous $\mathrm{SHH}$. However, no significant differences in proliferation were observed among the different intervention groups. Therefore, the activation of the SHH signaling pathway did not occur in an autocrine manner (31). Thus, the activation of SHH signaling pathway by HO-1 occurs through other mechanisms, as for example, via transcriptional activation or alternative pathways $(32,33)$. Unfortunately, in this study, the detailed mechanisms of SHH signaling pathway activation and their role in HO-1-induced PC cell proliferation were not investigated, and thus further research is required on this matter.

Our data demonstrated that treatment with Gem led to the pronounced growth inhibition of the PC cells with the downregulation of HO-1. On the contrary, the upregulation of HO-1 suppressed the inhibitory effect of Gem on PC cell proliferation. Our in vitro results are similar to those of other studies on HO-1 or its metabolites and PC growth in vivo (4) and on $\mathrm{HO}-1$ and the responsiveness of $\mathrm{PC}$ to anticancer treatment (9). Furthermore, our results provide further information and add to the findings of these other studies, and the SHH pathway may be one of the mechanisms involved. Therefore, the specific inhibition of HO-1 expression may be used as a sensitizer to chemotherapy $(9,34)$.

In addition, we explored the potential mechanisms of HO-1-induced PC cell proliferation and the response of PC cells to Gem. Treatment of the PC cells with Gem strongly induced HO-1 expression. Moreover, Gem enhanced Gli1 expression, although no difference was observed in SHH expression. Thus, Gem is responsible for activation of the SHH pathway through Gli1, but not through autocrine SHH. The results revealed the combined antitumor effects of Gem and the SHH signaling pathway on PC cells (16). Although the relatively integrated analysis of the HO-1 mechanisms was carried out in this study, the actual cause and effect were not expounded completely, and thus further studies are warranted. However, the mechanisms of HO-1-induced cell proliferation are complex, and the results were shown only in vitro in this study. Thus, further studies performing in vivo experiments are also required.

In conclusion, the data from this study demonstrate that high HO-1 levels in PC cells are responsible for tumor cell proliferation and resistance to anticancer therapy. Furthermore, the SHH signaling pathway, which is initiated by HO-1, may act as an endogenous mechanism in this process. This study enhanced our understanding of the exact molecular processes of HO-1 and SHH in PC cells, may aid in the development of novel therapeutic targets for the treatment of patients with PC.

\section{Acknowledgements}

Not applicable.

\section{Funding}

This study was supported by the National Natural Science Foundation of China (grant no. 81502074).

\section{Availability of data and materials}

The analyzed data sets generated during the study are available from the corresponding author on reasonable request.

\section{Authors' contributions}

The author contributions were as follows: Design of the experiments, ZWa and LH; performing the experiments, LH and JJ; data analysis, $\mathrm{ZWu}$ and $\mathrm{QM}$; contribution of reagents/materials/analysis tools, ZWa; writing of the article, LH and JJ. All authors have read and approved the final manuscript.

\section{Ethics approval and consent to participate}

Not applicable.

\section{Consent for publication}

Not applicable.

\section{Competing interests}

The authors declare that they have no competing interests.

\section{References}

1. Siegel RL, Miller KD and Jemal A: Cancer Statistics, 2017. CA Cancer J Clin 67: 7-30, 2017.

2. Liu Q, Liao Q and Zhao Y: Chemotherapy and tumor microenvironment of pancreatic cancer. Cancer Cell Int 17: 68, 2017.

3. Otterbein LE and Choi AM: Heme oxygenase: Colors of defense against cellular stress. Am J Physiol Lung Cell Mol Physiol 279: L1029-L1037, 2000.

4. Nuhn P, Künzli BM, Hennig R, Mitkus T, Ramanauskas T, Nobiling R, Meuer SC, Friess $\mathrm{H}$ and Berberat PO: Heme oxygenase-1 and its metabolites affect pancreatic tumor growth in vivo. Mol Cancer 8: 37, 2009.

5. Heeba GH, Hamza AA and Hassanin SO: Induction of heme oxygenase-1 with hemin alleviates cisplatin-induced reproductive toxicity in male rats and enhances its cytotoxicity in prostate cancer cell line. Toxicol Lett 264: 38-50, 2016.

6. Jo EJ, Park SJ and Kim BC: Propyl gallate sensitizes human lung cancer cells to cisplatin-induced apoptosis by targeting heme oxygenase-1 for TRC8-mediated degradation. Eur J Pharmacol 788: 321-327, 2016.

7. Jang HJ, Hong EM, Kim M, Kim JH, Jang J, Park SW, Byun HW, Koh DH, Choi MH, Kae SH, et al: Simvastatin induces heme oxygenase-1 via NF-E2-related factor 2 (Nrf2) activation through ERK and PI3K/Akt pathway in colon cancer. Oncotarget 7: 46219-46229, 2016.

8. Schwer CI, Guerrero AM, Humar M, Roesslein M, Goebel U, Stoll P, Geiger KK, Pannen BH, Hoetzel A and Schmidt R: Heme oxygenase-1 inhibits the proliferation of pancreatic stellate cells by repression of the extracellular signal-regulated kinase $1 / 2$ pathway. J Pharmacol Exp Ther 327: 863-871, 2008.

9. Berberat PO, Dambrauskas Z, Gulbinas A, Giese T, Giese N, Künzli B, Autschbach F, Meuer S, Büchler MW and Friess H: Inhibition of heme oxygenase-1 increases responsiveness of pancreatic cancer cells to anticancer treatment. Clin Cancer Res 11: 3790-3798, 2005.

10. Yoo YA, Kang MH, Lee HJ, Kim BH, Park JK, Kim HK, Kim JS and Oh SC: Sonic hedgehog pathway promotes metastasis and lymphangiogenesis via activation of Akt, EMT, and MMP-9 pathway in gastric cancer. Cancer Res 71: 7061-7070, 2011. 
11. Han L, Ma J, Duan W, Zhang L, Yu S, Xu Q, Lei J, Li X, Wang Z, Wu Z, et al: Pancreatic stellate cells contribute pancreatic cancer pain via activation of sHH signaling pathway. Oncotarget 7: 18146-18158, 2016.

12. Jung IH, Jung DE, Park YN, Song SY and Park SW: Aberrant Hedgehog ligands induce progressive pancreatic fibrosis by paracrine activation of myofibroblasts and ductular cells in transgenic zebrafish. PLoS One 6: e27941, 2011.

13. Shukla SK, Purohit V, Mehla K, Gunda V, Chaika NV, Vernucci E, King RJ, Abrego J, Goode GD, Dasgupta A, et al: MUC1 and HIF-1alpha signaling crosstalk induces anabolic glucose metabolism to impart gemcitabine resistance to pancreatic cancer. Cancer Cell 32: 392, 2017.

14. Jin X, Pan Y, Wang L, Ma T, Zhang L, Tang AH, Billadeau DD, Wu H and Huang H: Fructose-1,6-bisphosphatase inhibits ERK activation and bypasses gemcitabine resistance in pancreatic cancer by blocking IQGAP1-MAPK interaction. Cancer Res 77: 4328-4341, 2017.

15. Wang H, Ning Z, Li Y, Zhu X and Meng Z: Bufalin suppresses cancer stem-like cells in gemcitabine-resistant pancreatic cancer cells via Hedgehog signaling. Mol Med Rep 14: 1907-1914, 2016.

16. Kim EJ, Sahai V, Abel EV, Griffith KA, Greenson JK, Takebe N, Khan GN, Blau JL, Craig R, Balis UG, et al: Pilot clinical trial of hedgehog pathway inhibitor GDC-0449 (vismodegib) in combination with gemcitabine in patients with metastatic pancreatic adenocarcinoma. Clin Cancer Res 20: 5937-5945, 2014.

17. Livak KJ and Schmittgen TD: Analysis of relative gene expression data using real-time quantitative PCR and the 2(-Delta Delta C(T)) method. Methods 25: 402-408, 2001

18. Cao Z, Geng B, Xu S, Xuan W, Nie L, Shen W, Liang Y and Guan R: BnHO1, a haem oxygenase-1 gene from Brassica napus, is required for salinity and osmotic stress-induced lateral root formation. J Exp Bot 62: 4675-4689, 2011.

19. Chen R, Lai LA, Sullivan Y, Wong M, Wang L, Riddell J, Jung L, Pillarisetty VG, Brentnall TA and Pan S: Disrupting glutamine metabolic pathways to sensitize gemcitabine-resistant pancreatic cancer. Sci Rep 7: 7950, 2017.

20. Sunamura M, Duda DG, Ghattas MH, Lozonschi L, Motoi F, Yamauchi J, Matsuno S, Shibahara S and Abraham NG: Heme oxygenase-1 accelerates tumor angiogenesis of human pancreatic cancer. Angiogenesis 6: 15-24, 2003.

21. Jakstaite A, Maziukiene A, Silkuniene G, Kmieliute K, Gulbinas A and Dambrauskas Z: HuR mediated post-transcriptional regulation as a new potential adjuvant therapeutic target in chemotherapy for pancreatic cancer. World J Gastroenterol 21: 13004-13019, 2015.
22. Bai Y, Bai Y, Dong J, Li Q, Jin Y, Chen B and Zhou M: Hedgehog signaling in pancreatic fibrosis and cancer. Medicine (Baltimore) 95: e2996, 2016.

23. Lee RT, Zhao Z and Ingham PW: Hedgehog signalling. Development 143: 367-372, 2016.

24. Tadros S, Shukla SK, King RJ, Gunda V, Vernucci E, Abrego J, Chaika NV, Yu F, Lazenby AJ, Berim L, et al: De novo lipid synthesis facilitates gemcitabine resistance through endoplasmic reticulum stress in pancreatic cancer. Cancer Res 77: 5503-5517, 2017.

25. Pishvaian MJ and Brody JR: Therapeutic implications of molecular subtyping for pancreatic cancer. Oncology (Williston Park) 31: 159-166, 168, 2017.

26. Adamska A, Domenichini A and Falasca M: Pancreatic ductal adenocarcinoma: Current and evolving therapies. Int J Mol Sci 18: $18,2017$.

27. Borowa-Mazgaj B: Pancreatic cancer-mechanisms of chemoresistance. Postepy Hig Med Dosw 70: 169-179, 2016 (In Polish).

28. Güngör C, Hofmann BT, Wolters-Eisfeld G and Bockhorn M: Pancreatic cancer. Br J Pharmacol 171: 849-858, 2014.

29. Catenacci DV, Junttila MR, Karrison T, Bahary N, Horiba MN, Nattam SR, Marsh R, Wallace J, Kozloff M, Rajdev L, et al: Randomized phase Ib/II study of gemcitabine plus placebo or vismodegib, a hedgehog pathway inhibitor, in patients with metastatic pancreatic cancer. J Clin Oncol 33: 4284-4292, 2015.

30. Cao J, Peterson SJ, Sodhi K, Vanella L, Barbagallo I, Rodella LF, Schwartzman ML, Abraham NG and Kappas A: Heme oxygenase gene targeting to adipocytes attenuates adiposity and vascular dysfunction in mice fed a high-fat diet. Hypertension 60: 467-475, 2012.

31. Felley-Bosco E, Opitz I and Meerang M: Hedgehog signaling in malignant pleural mesothelioma. Genes (Basel) 6: 500-511, 2015.

32. Wendling-Keim DS, Wanie L, von Schweinitz D, Grantzow R and Kappler R: Transcriptional activation of Hedgehog pathway components in aggressive haemangioma. Exp Dermatol 26: 934-939, 2017.

33. Huang H, Cotton JL, Wang Y, Rajurkar M, Zhu LJ, Lewis BC and Mao J: Specific requirement of Gli transcription factors in Hedgehog-mediated intestinal development. J Biol Chem 288: 17589-17596, 2013.

34. Frank J, Lornejad-Schäfer MR, Schöffl H, Flaccus A, Lambert C and Biesalski HK: Inhibition of heme oxygenase-1 increases responsiveness of melanoma cells to ALA-based photodynamic therapy. Int J Oncol 31: 1539-1545, 2007. 